Supplement of Nonlin. Processes Geophys., 27, 1-9, 2020

https://doi.org/10.5194/npg-27-1-2020-supplement

(C) Author(s) 2020. This work is distributed under

the Creative Commons Attribution 4.0 License.

(c) (1)

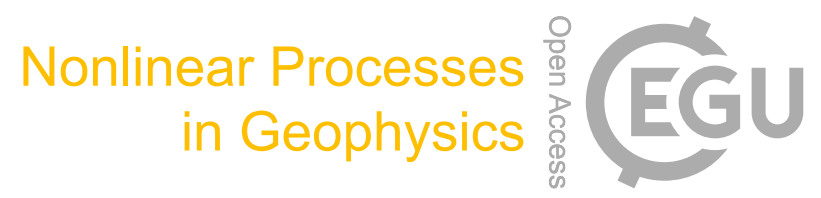

Supplement of

\title{
Magnitude correlations in a self-similar aftershock rates model of seismicity
}

\section{Andres F. Zambrano Moreno and Jörn Davidsen}

Correspondence to: Andres F. Zambrano Moreno (andres.zambranomoren@ucalgary.ca)

The copyright of individual parts of the supplement might differ from the CC BY 4.0 License. 


\section{Randomizing methodology}

This supplemental material is part of Sec. 4 in (Zambrano Moreno, 2019). Our study of magnitude correlations, similar to methods used in (Lippiello et al., 2008; Davidsen and Green, 2011), was done by considering subsequent events in the time ordered catalog; $\Delta m_{i}=m_{i+1}-m_{i}$ (for a particular magnitude threshold; $m_{t h}$ ) and then compared these to randomized magnitude differences averaged over 500 realizations; $\Delta m_{i}^{*}=m_{i^{*}}-m_{i}\left(m_{i^{*}}\right.$ being a magnitude chosen at random). The comparison was done by means of the difference between the CDFs, $P(\ldots)$, of $\Delta m_{i}$ and $\Delta m_{i}^{*}$. To obtain the magnitude differences between subsequent events we divide the catalog into two lists $\left(L_{1}\right.$ and $\left.L_{2}\right)$ as shown in Fig. 1. Each list now forms a column that is arranged in the form shown in the left most matrix of Fig. 2 (for simplicity only indexes are shown) where we take the difference between the magnitudes of each row $\left(\Delta m_{i}=m_{i+1}-m_{i}\right)$ to obtain a single list $\Delta m=L_{2}-L_{1}$. Similarly, we then randomize the $L_{2}$ column (the subsequent events) $N$ times and calculate the corresponding lists: $\Delta m^{1 *}=L_{2}-L_{1}, \Delta m^{2 *}=L_{2}-L_{1}$ up to $\Delta m^{N *}=L_{2}-L_{1}=m_{i}^{N *}-m_{i}$ (where the red color for $L_{2}$ represents the randomized list).

To obtain the CDF for the unrandomized subsequent magnitude differences $(\Delta m)$ we test whether any event pair falls below a particular magnitude value $m_{0}$, where those that do are kept in the list and the CDF (see left matrix Fig. 3) for this particular $m_{0}$ is calculated (the $m_{0}$ values lie in the range $[-4.00, \ldots, 4.00]$ for our analysis). In a similar fashion, for each of the randomized cases we obtain the particular $\mathrm{CDF}$ and then calculate the mean in order to obtain the mean randomized CDF (right three matrices in Fig. 3). These last two procedures allows us to obtain the quantity $\delta P \equiv P\left(\Delta m<m_{0}\right)-P\left(\Delta m^{*}<m_{0}\right)$. If magnitude correlations between subsequent events in the ordered catalog are present, the distribution of $\Delta m$ will deviate from the distribution of the randomized case; $\Delta m^{*}$. To assess whether magnitude correlations exist in the SSAR model we considered three types of conditioning for various $m_{t h}$ values:

$L_{2}\left\{\begin{array}{ccc}\text { Index } & \text { Time (s) } & \mathrm{m} \\ 0 & 0.00 & 2.1 \\ 1 & 12.36 & 1.3 \\ 2 & 100.23 & 3.5 \\ 3 & 110.55 & 2.9 \\ 4 & 115.22 & 1.0 \\ : & : & :\end{array}\right\} L_{1}$

Figure 1. Creating two lists $\left(L_{1}\right.$ and $\left.L_{2}\right)$ from a single catalog is achieved by 'shifting' rows in order to create two lists. In our particular case, $L_{2}$ corresponds to the subsequent events. 


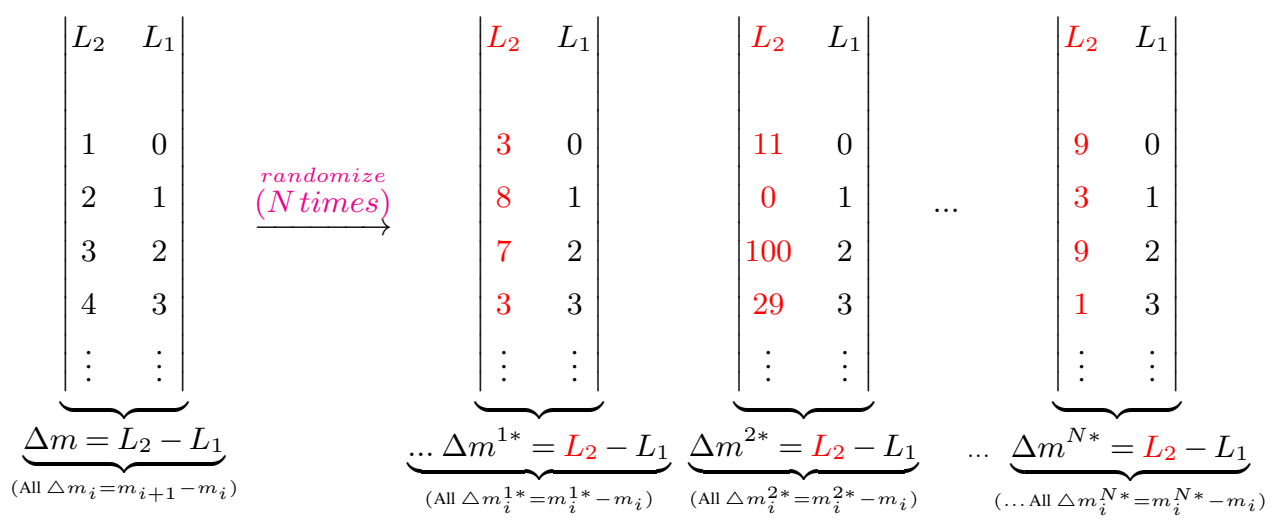

Figure 2. Arrangement of lists $L_{1}$ and $L_{2}$. The difference between each row of $L_{2}$ and $L_{1}$ gives the $\Delta m_{i} \mathrm{~s}$ and $\Delta m_{i}^{*} \mathrm{~s}$ for the original lists (left) and the $N$ randomizations of $L_{2}$ (right 3 matrices). For clarity only indexes are shown.

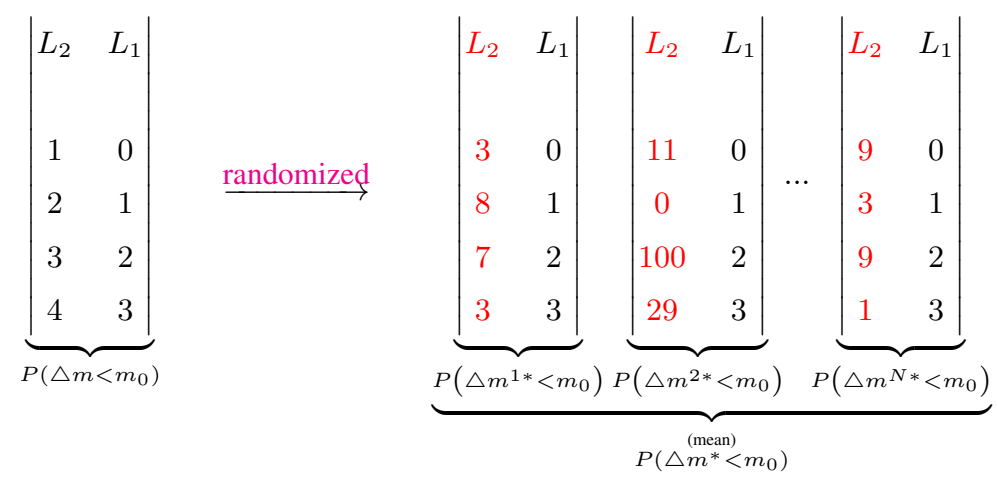

Figure 3. Description of the procedure to obtain the CDF for the unrandomized and randomized catalogs. For a particular value of $m_{0}$ : in the unrandomized list $\Delta m$ one keeps all event pairs that are bellow $m_{0}$ and then calculates the CDF, for the randomized case we first calculate the distribution for each randomized catalog and then take the mean of the $N$ CDFs. Red color for $L_{2}$ represents the randomized list.

- unconditioned; there is no condition in time or triggering relation for subsequent events (one essentially takes the whole catalog), one takes all subsequent event pairs $\Delta m_{i}$,

- $\Delta t$; one only considers the subsequent event pairs $\Delta m_{i}$ whose time difference is below the time interval $\Delta t$,

- $\Delta t \&$ M-D (mother-daughter); one only considers subsequent event pairs $\Delta m_{i}$ that fall below the time interval $\Delta t$ and are also a mother-daughter pair,

- only M-D; one considers subsequent event pairs $\Delta m_{i}$ that are a mother-daughter pair.

The description provided above and in Fig. 3 applies to what we call the unconditioned case where we use the quantity $5 \delta P\left(m_{0}\right) \equiv P\left(\Delta m<m_{0}\right)-P\left(\Delta m^{*}<m_{0}\right)$, which corresponds to the difference of the CDF for the ordered and randomized catalog, respectively $\left(-4.00<m_{0}<4.00\right)$. Similarly we can also condition subsequent event pairs: for $\Delta t$ and $\Delta t \&$ M-D 
conditioning we have the respective quantities;

$\delta P\left(m_{0} \mid \Delta t<y\right)=P\left(\Delta m<m_{0} \mid \Delta t<y\right)-P\left(\Delta m^{*}<m_{0} \mid \Delta t<y\right)$,

and

$\delta P\left(m_{0} \mid \Delta t<y \& \mathrm{M}-\mathrm{D}\right)=P\left(\Delta m<m_{0} \mid \Delta t<y \& \mathrm{M}-\mathrm{D}\right)-P\left(\Delta m^{*}<m_{0} \mid \Delta t<y \& \mathrm{M}-\mathrm{D}\right)$.

When $y$ is equal to the period of the catalog then we obtain the case of only M-D conditioning: $\delta P\left(m_{0} \mid\right.$ M-D $)$.

The reason why we choose to condition on time when M-D conditioning is motivated by the hypothesis that one would expect events that are closer in time are more likely to be correlated than those further apart (in the model all the correlations by construction are at the mother-daughter level, viz. Eq. 2 in the main text). By conditioning on M-D and time we are also 'picking' certain magnitude differences via the rate equation, Eq. 2 in the main text. Another important aspect in our analysis is how we randomly choose the magnitudes $m_{i^{*}}$ in the case of $\Delta t$ or $\Delta t \&$ M-D conditioning; one can either pick $m_{i^{*}}$ 's from the already conditioned catalog (sub-catalog randomizing) or one can pick $m_{i^{*}}$ 's from the full unconditioned catalog (full-catalog randomizing). In sub-catalog randomizing we do not change the frequency-magnitude distribution (FMD) for the $m_{i^{*}}$ 's since we are only picking magnitudes from a list of $m_{i}^{\prime}$ s which have satisfied the conditioning.

When $\Delta t \&$ M-D conditioning, the two randomizing methods used in our analysis, one which keeps the FMD fixed while the other does not, both produce different types of magnitude correlations. Specifically, they differ in that when the frequencymagnitude distribution is fixed we are seeing the inherent (non-trivial) magnitude correlations, while the correlations in the other case (trivial) can arise due to variations in the FMD. More precisely, when we consider only $\Delta t \&$ M-D conditioning for a particular $m_{t h}$, the conditioned event pairs will satisfy the FMD dictated by Eq. 7 in the main text: when sub-catalog randomizing, one picks $m^{*}$ 's that come from this particular FMD, whereas in full-catalog randomizing one can pick $m^{*}$ 's from the full unconditioned catalog and thus the overall FMD is accessible. As a specific example, in Fig. 4 we show the FMD for the two types of randomization applied to a catalog of the SSAR model for $\Delta t \&$ M-D conditioning and $2.2<m_{i}<3.0$.

For all three types of conditioning one can state the following; if there are correlations between subsequent magnitudes one would expect the quantity $\delta P\left(m_{0}\right)$ to significantly deviate from 0 for any value of $m_{0}$. 

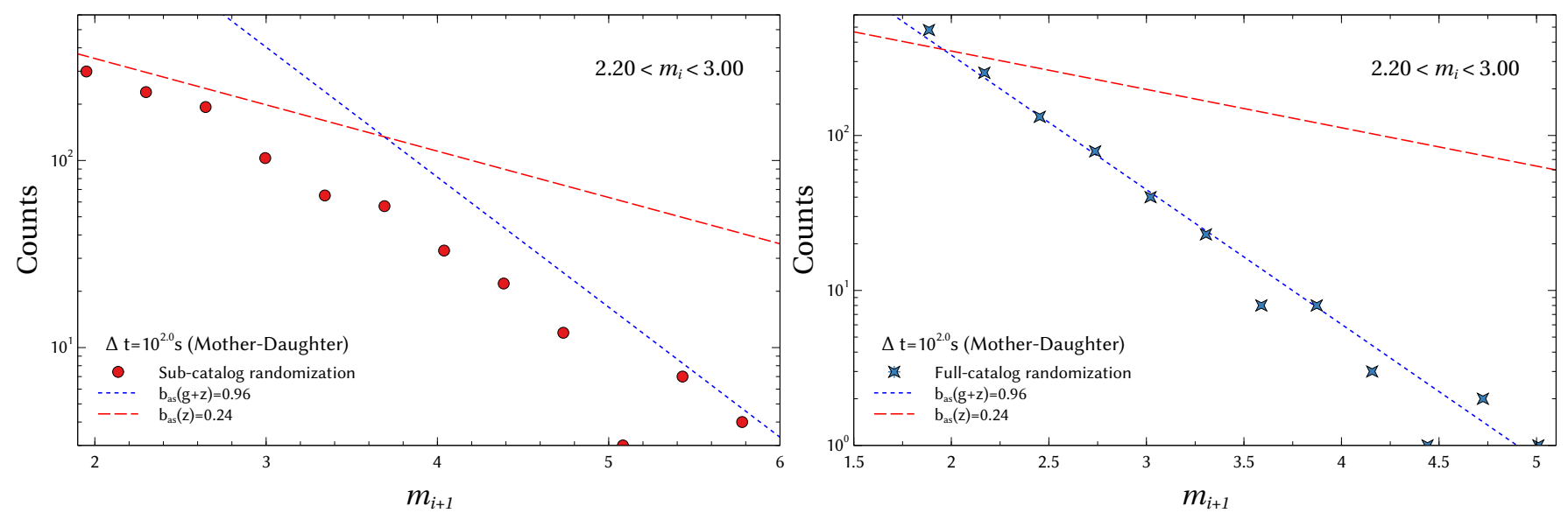

Figure 4. Plots of the FMD for: sub-catalog (left) and full-catalog (right) randomizing using ' $\Delta t \&$ M-D conditioning. A short interval for the $m_{i}$ 's was chosen in order to plot the FMD.

\section{References}

Davidsen, J. and Green, A.: Are Earthquake Magnitudes Clustered?, Physical Review Letters, 106, https://doi.org/10.1103/PhysRevLett.106.108502, 2011.

Lippiello, E., de Arcangelis, L., and Godano, C.: Influence of Time and Space Correlations on Earthquake Magnitude, Physical Review

Letters, 100, 038 501, https://doi.org/10.1103/PhysRevLett.100.038501, 2008.

Zambrano Moreno, A. F.: Magnitude correlations and criticality in a self-similar model of seismicity, PRISM Dataverse (University of Calgary), 1, 184, https://doi.org/hdl.handle.net/1880/110243, 2019. 